\title{
A Fuzzy Method for Assessing Eco-Environmental Disaster Risk Caused by Coalbed Methane in China
}

\author{
Ye Xue ${ }^{1,2, *}$, Xiaoxiao Li $i^{1}$, Wen Sun ${ }^{1}$, Baozhang Chen ${ }^{2}$ \\ ${ }^{1}$ College of Economics and Management, Taiyuan University of Technology, Taiyuan 030024, China \\ ${ }^{2}$ Institute of Geographic Sciences and Natural Resources Research, Chinese Academy of Sciences, \\ Beijing 100101, China
}

Received September 29, 2017

Accepted January 8, 2018

\begin{abstract}
Based on an improved risk assessment index system, this paper constructs an eco-environmental disaster risk assessment model during coalbed methane industrialization development in China by using intuitionistic fuzzy sets to describe the uncertain risk information, and the transformed interval value of Mamdani intuitionistic fuzzy neural networks model. Then, the validity of the model is verified by simulation tests. Furthermore, the assessment results are compared with those obtained by fuzzy neural networks model. Results show that suggested model has multidimensional nonlinearity and global approximation characteristics. By the procedure of "fuzzification fuzzy-rules - defuzzifier", the output conversion from uncertainty quantitative indicators to accurate risk assessment values can be effectively realized. Compared with the fuzzy neural networks model, the suggested model has better accuracy and stability. The risk assessment value calculated by the suggested model fairly matches the expected one. The study supplies a decision support for routine supervision and risk precaution and management on one hand, and enriches the theoretical research of the eco-environmental risk assessment of coalbed methane industrialization development on the other hand.
\end{abstract}

Keywords: Coalbed methane; eco-environment; risk assessment; intuitionistic fuzzy set; fuzzy neural system

\section{Introduction}

Coalbed methane (CBM) is one of the most reliable supplements to oil and gas. However, with the rapid increase of CBM production, regional ecological risk problems become increasingly prominent. Let's take the eco-environmental deterioration issue in Qinshui basin, Shanxi province of China as illustration. Wang (2014) conducted an indoor experiment of soil column simulated irrigation for a long time and finally drawn the conclusions: the produced water of South Shizhuang CBM test area was with high-salinity, high-chloride and alkalinity characteristics, and the soil salinity increased, water retention capacity declined after a long-time irrigation, which resulted in massive crop failures. Liu et al. (2014) selected water-formation samples, reservoir hydrops samples, bottom sediment samples of Fanzhuang area for test, and confirmed that the heavy metal content of arsenic, cadmium, stannum, etc. in produced water is high. Meanwhile, the topsoil around CBM wells were confronted with the contamination risk of heavy mentals when the accumulation, migration and enrichment of such kinds of chemical elements happened. Hu et al.
(2009) insisted that during the process of CBM production, a large amount of draining water from coal seams would lead to the contamination of surface water, the decline of groundwater level, the disruption of aquatic ecological environment, and the denudation of soil. Li et al. (2014) investigated the region of Zhengzhuang and suggested that the severely supracrustal and hypogeal disturbance during the construction and production period of $\mathrm{CBM}$ industry made great changes on regional underlying topography, which may result in the disruption of vegetation, the newly arose soil erosion, and the damage of climatope. Besides, since the dominant component of $\mathrm{CBM}$ is methane $\left(\mathrm{CH}_{4}\right)$, the greenhouse effect is 22 times over carbon dioxide $\left(\mathrm{CO}_{2}\right)$ (Song et al., 2012), and the leak of CBM may bring the conditions of safety production and ecological civilization into the grave threat. Therefore, we should strength the risk management of ecological environment on CBM industrialization development, whose premise is to reasonably and reliably assess such risks by quantitative approaches.

America and Canada early carried out the study the issues of the eco-environmental risk assessment on CBM exploitation. In the late twentieth century, Beckstrom and 
Boyer (1993) analyzed the aquifer-protection works on CBM producing wells and deep cathodic-protection groundbeds. Chafin et al. (1996) studied the effect of hydrofracture and the degradation of groundwater level on eco-environmental risk. U.S. Environmental Protection Agency (EPA) evaluated the impact of the hydrofracture of CBM reservoirs on the drinking water resource by stages, and respectively from three aspects: actual observation records, experimental data, and theoretical analysis. Cheung et al. $(2009,2010)$ assessed the impact of the geochemistry of trace metals and rare earth elements in produced fluids or shallow groundwater in CBM producing regions of Alberta, Canada. Dahm et al. (2013) utilized 3-D fluorescence spectroscopy to distinguish the naturally occurring organic from anthropogenic chemical compounds in the CBM produced water in order to recognize the risk of aquifer contamination. Aguiler et al. (2014) analyzed the effect of "resource endowments, economics and the environment" in conventional, tight gas, shale and coalbed methane reservoirs.

The studies in China mainly took qualitative methods, and the quantitative approaches are relatively few. For instance, Pei et al. (2015) applied the evaluation model of pressure-state-response (PSR) to construct the framework and index system of CBM ecological system. Sun et al. (2015) set up an index system of ecological risk assessment on CBM industrialization development, based on the logic framework "Hazard-induced environment Caused-hazards - Hazard-affected bodies - Disaster losses". Nonetheless, there is a lack of comprehensive and effective quantitative evaluation models. The reason is twofold. On one hand, it is hard to acquire the actual data, and there is much uncertainty in the obtained data information. As a result, the specialized database has not been set up that can efficaciously link among the environmental data from different regions. On the other hand, it is due to the lack of the unified evaluation index system and valid quantitative criteria. Therefore, this paper plans to screen and optimize the index system proposed by Sun et al. (2015), and then improve the quality of data processing with uncertain risk information by means of the intuitionistic fuzzy numbers transformed by interval values. Ultimately, based on the Mandani intuitionistic fuzzy neural network, an eco-environmental risk assessment model for the CBM industrialization development is constructed.

\section{Optimization of Eco-environmental Risk}

\section{Assessment Index System}

Sun and Xue (2015) constructed an eco-environmental risk assessment index system of CBM industrialization development based on the theory of disaster system, and defined the eco-environmental risk of CBM, $R_{C B M}$, as the set \{hazard-induced environment, caused-hazards, hazard-affected bodies, disaster losses\}, where the stability of hazard-induced environment $(E)$, the risk of caused-hazards ( $C$ ), and the vulnerability of hazard-affected bodies $(B)$ are defined to represent the probability of eco-environmental risk while the damage of disaster losses $(L)$ are designed to reflect the probability of risk losses. The combination of them constitutes the eco-environmental risk of $\mathrm{CBM}$ industrialization development.

Based on this model, the paper makes the following modifications:

(1) Transform some indexes into relative ones. For instance, use "proportion of economic loss" instead of "economic loss". In doing so, the indexes become comparable and the calculation is simplified.

(2) Optimize the exposure level, adaptive capacity and anti-risk capability of hazard-affected bodies from three aspects: personnel, property and materials. For instance, in considering that with the increase of CBM resource exploration, the more hazard-affected bodies $(B)$ are exposed in ecological risk and thus the greater probability of the risk could happen. Hence, the index "resource exposure degree of CBM" is added.

(3) Specialize the artificial caused-hazard in caused-hazards. For instance, to assess the groundwater pollution and soil pollution, we can specialize in the impact of comprehensive hazard behaviors by means of "standard evaluation index of major groundwater pollution factors" and "Nemero pollution index of soil".

Based on the theory of rough set and Rosetta software, the paper finally determines an index system by screening and optimizing evaluation indexes, which is shown in Table 1.

\section{Quantification of Risk Assessment Indexes}

\subsection{Fuzzification method}

As shown in Table 1, the ecological risk information itself has uncertainty in general. For example, in the practical 
Table 1. Eco-environmental Risk Assessment Index System of CBM Industrialization Development

\begin{tabular}{|c|c|c|}
\hline First level index & $\begin{array}{l}\text { Second level } \\
\text { indexes }\end{array}$ & Third level indexes \\
\hline \multirow{6}{*}{ Eco-environmental risk } & \multirow{3}{*}{$\begin{array}{c}\text { The stability of } \\
\text { hazard-induced } \\
\text { environment } \\
E\end{array}$} & $e_{1}$ complexity of the hydrogeological condition \\
\hline & & $e_{2}$ mechanization degree \\
\hline & & $e_{3}$ reliability of mechanical equipment \\
\hline & \multirow{5}{*}{$\begin{array}{c}\text { The risk of } \\
\text { caused-hazards } \\
C\end{array}$} & $c_{1}$ impact of bad weather \\
\hline & & $\begin{array}{l}c_{2} \text { ratio of maximum ground-level concentration } \\
\text { to standard concentration of major air pollutants }\end{array}$ \\
\hline & & $\begin{array}{l}c_{3} \text { standard evaluation index of groundwater } \\
\text { major pollution factors }\end{array}$ \\
\hline \multirow{8}{*}{$\begin{array}{c}\text { industrialization } \\
\text { development }\end{array}$} & & $c_{4} \quad$ Nemero pollution index of soil \\
\hline & & $c_{5}$ percentage of forestry and grass coverage \\
\hline & \multirow{3}{*}{$\begin{array}{c}\text { The vulnerability of } \\
\text { hazard-affected } \\
\text { bodies } \\
B\end{array}$} & $b_{1}$ resource exposure degree of $\mathrm{CBM}$ \\
\hline & & $b_{2}$ ability of personal risk prevention \\
\hline & & $\begin{array}{l}b_{3} \text { investment proportion of prevention } \\
\text { construction funds }\end{array}$ \\
\hline & \multirow{3}{*}{$\begin{array}{c}\text { The damage of } \\
\text { disaster losses } \\
L\end{array}$} & $l_{1}$ proportion of economic loss \\
\hline & & $l_{2}$ incidence of occupational diseases \\
\hline & & $l_{3}$ casualty rate \\
\hline
\end{tabular}

risk assessment process, we may frequently encounter the situation that experts are hesitant in their judgments over risk information, or that experimenters are hard to obtain the data precisely due to the systematic error. With the emergence of intuitionistic fuzzy sets theory (Atanassov, 1986), the nonmembership functions and hesitancy degree functions are added to general fuzzy numbers, which improves the quality of data processing and realizes the precise expression of fuzzy uncertainty information. The definition of an intuitionistic fuzzy set is as follows:

Definition 1 Let $X$ be a given universe of discourse, and an intuitionistic fuzzy set is defined by

$$
\tilde{A}(x)=\left\{<x, \mu_{\tilde{A}}(x), v_{\tilde{A}}(x)>\mid x \in X\right\}
$$

where $\mu_{\tilde{A}}(x)$ and $v_{\tilde{A}}(x)$ are respectively the membership function and non-membership function of set $\widetilde{A}$, which respectively means the degree to which $x$ belongs to $\widetilde{A}$ and the degree to which $x$ does not belong to $\widetilde{A}$ such that $0 \leq \mu_{\tilde{A}}(x) \leq 1, \quad 0 \leq v_{\tilde{A}}(x) \leq 1 \quad$ and $\quad 0 \leq \mu_{\tilde{A}}(x)+$ $v_{\tilde{y}}(x) \leq 1$. In addition, the hesitancy degree function is $\pi_{\tilde{A}}(x)=1-\mu_{\tilde{A}}(x)-v_{\tilde{A}}(x)$, and the smaller value corresponds to the more certain risk information.

An intuitionistic fuzzy set is equivalent to an interval-valued fuzzy set (Yuan 2013). Considering the inequality $\mu_{\tilde{A}}(x) \leq 1-v_{\tilde{A}}(x)$, the intuitionistic fuzzy set $\widetilde{A}$ can be transformed into an interval-valued fuzzy set using the following formula:

$$
\tilde{A}(x)=\left(\mu_{\tilde{A}}, v_{\tilde{A}}\right) \mapsto \tilde{A}(x)=\left[\mu_{\tilde{A}}, 1-v_{\tilde{A}}\right]
$$

The membership function $\mu_{\tilde{A}}(x)$ can be of any type including Gaussian, Sigmoid or Triangular function etc. We select the Gaussian membership function in this paper in considering that the function has high smoothness. The Gaussian membership function is defined by and lower bounds of the interval-valued fuzzy numbers respectively as shown in Fig. 1.

$$
\mu_{\tilde{A}}(x)=f(x, \sigma, \delta)=\exp \left(-\left(\frac{x-\delta}{\sigma}\right)^{2}\right)
$$

where $\delta$ and $\sigma$ represent respectively the curve center and curve width of the Gaussian membership function.

Substituting (3) into (2), and combining with 
the features of Gaussian function, intuitionistic fuzzy numbers can be transformed into interval valued fuzzy numbers with the same

\section{fuzzified.}

(1) Complexity $\widetilde{e}_{1}$ of the hydrogeological

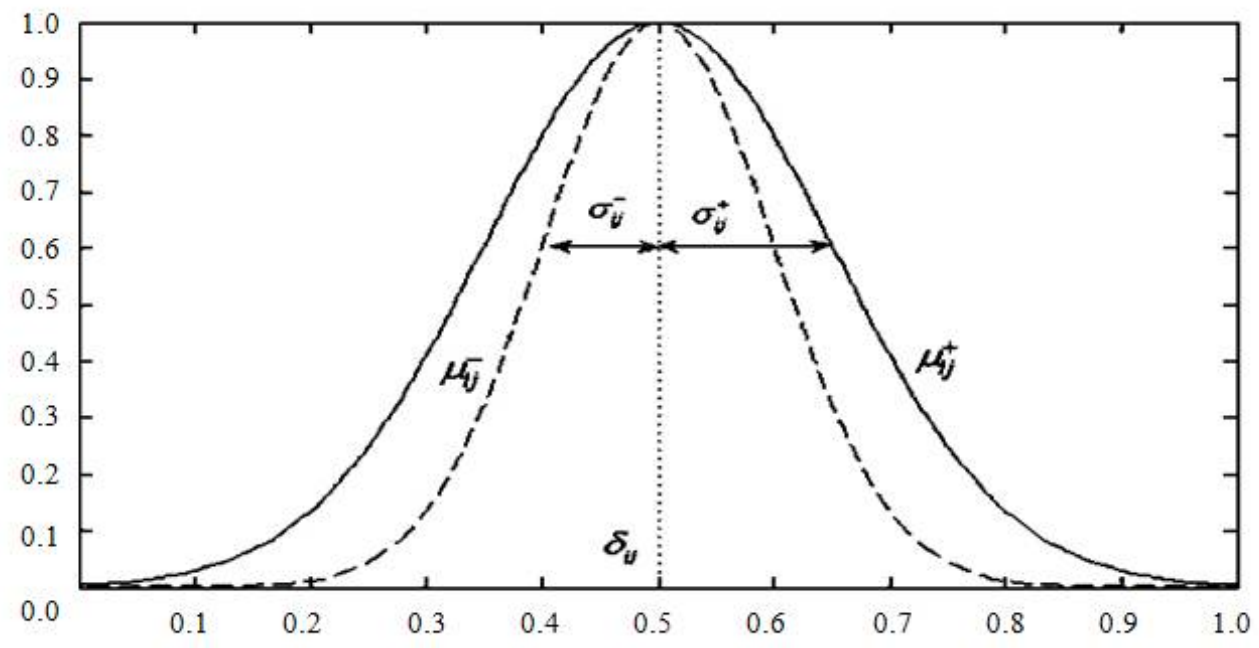

Fig. 1. Transformation of a Gaussian Intuitionistic Fuzzy Number into an Interval-valued Fuzzy Number.

Table 2. "IF-THEN" Risk Decision Rules

\begin{tabular}{cccc}
\hline & Input parameters & & Output parameters \\
Coal seam & Aquifer & Groundwater & Hydrogeological condition \\
stability & vulnerability & sensitivity & complexity \\
\hline instable & vulnerable & sensitive & very complex \\
instable & vulnerable & sensitive & very complex \\
$\vdots$ & $\vdots$ & $\vdots$ & $\vdots$ \\
stable & invulnerable & insensitive & very simple \\
\hline
\end{tabular}

curve center $\delta_{i j}$ and different curve widths $\sigma_{i j}$, as shown through the following (4) to (6):

$$
\begin{gathered}
\widetilde{A}\left(x_{i}\right)=\left[\mu_{i j}^{-}\left(x_{i}\right), \mu_{i j}^{+}\left(x_{i}\right)\right] \\
\mu_{i j}^{-}\left(x_{i}\right)=\exp \left[-\left(\frac{x_{i}-\delta_{i j}}{\sigma_{i j}^{-}}\right)^{2}\right], i=1,2 \cdots n ; j=1,2 \cdots m(5) \\
\mu_{i j}^{+}\left(x_{i}\right)=\exp \left[-\left(\frac{x_{i}-\delta_{i j}}{\sigma_{i j}^{+}}\right)^{2}\right], i=1,2 \cdots n ; j=1,2 \cdots m(6)
\end{gathered}
$$

where $\mu_{i j}^{+}\left(x_{i}\right)$ and $\mu_{i j}^{-}\left(x_{i}\right)$ denote the upper

In this way, fuzzy uncertainty information is theoretically expressed as Gaussian intuitionistic fuzzy number so that the actual expression of fuzzy information is realized.

\subsection{Fuzzification of risk evaluation indexes}

Following the method of fuzzification method in Section 3.1, the indexes from Table 1 can be condition is a key index of natural hazard-induced environment, which is determined by three factors: the stability of coal seam, the vulnerability of aquifer and the sensibility of groundwater environment. For its fuzzification, we refer to "IF-THEN" risk decision rules (Xue 2014). Furthermore, we can obtain five output parameter values: very simple, simple, general, complex, very complex after the experts input the fuzzy linguistic variables of the above three factors as shown in Table 2 and Fig. 2. By Section 3.1, $\widetilde{e}_{1}$ is an intuitionistic fuzzy number with the Gaussian membership function.

(2) Mechanization degree $\widetilde{e}_{2}$ is determined by the utilization rate of mechanical equipments, the mine integrated unit yield of CBM and the informationization degree of management (Xie et al, 2012). $\widetilde{e}_{2}$ can be fuzzified by means of "IF-THEN" rules presented in Table 2. and Fig. 2. 


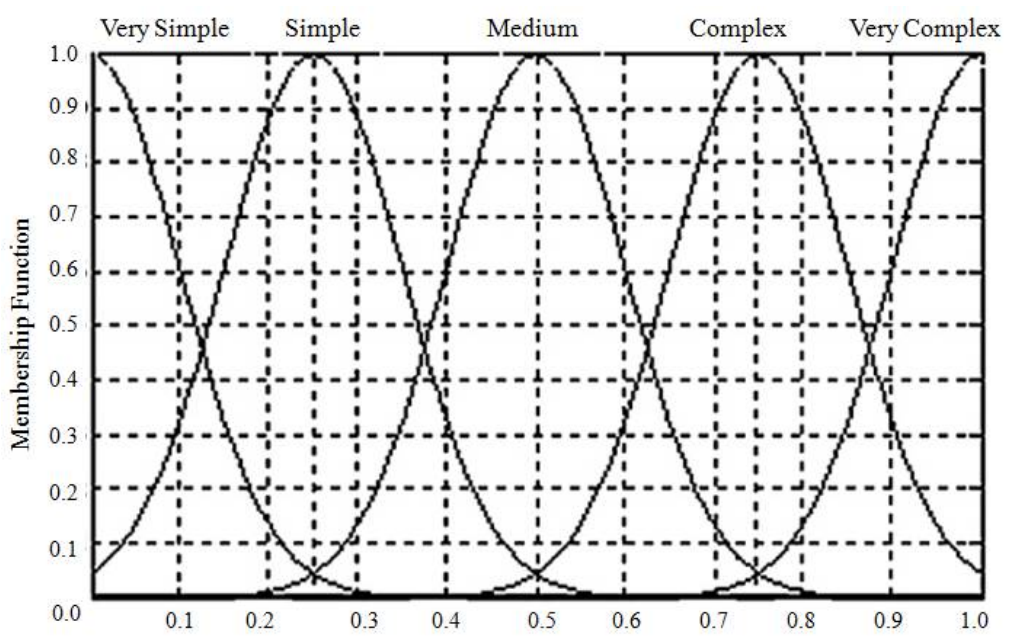

Fig. 2. The Complexity $\widetilde{e}_{1}$ of Hydrogeological Conditions.

(3) Reliability $\widetilde{e}_{3}$ of mechanical equipmen -ts is another key index of anthropogenic hazardinduced environment. We focus on the security attribute, i.e. the ability with which mechanical equipments can prevent from the environmental disruption, casualties and property loss. $\widetilde{e}_{3}$ could be readily calculated by the following:

$$
\tilde{e}_{3}=\exp \left(-\tilde{\lambda}_{s} \cdot \tilde{t}_{m}\right)
$$

where $\tilde{\lambda}_{s}$ is the fuzzy failure rate of the equipments or the system which may cause the contamination accident, and $\widetilde{t}_{m}$ is the fuzzy time of fulfilling CBM productive tasks.

(4) Impact degree $\widetilde{c}_{1}$ of bad weather is determined by three factors: the damage degree \{strong, general, weak\}, the time of duration \{long, medium, short $\}$ and the affected areas \{large, medium, small\}. $\widetilde{c}_{1}$ can be estimated by experts, and fuzzified in the same way as $\widetilde{e}_{1}$.

(5) Ratio $\widetilde{c}_{2}$ of maximum ground-level concentration to standard concentration of major air pollutants is used for evaluating the impact of atmospheric environment. Usually, 1 to 3 major pollutants are chosen and the ratio of each pollutant is calculated, and $\widetilde{c}_{2}$ is the fuzzification of the maximum ratio:

$$
\tilde{c}_{2}=\max _{m=1}^{3} \tilde{C}_{m}\left(C_{0 m}\right)^{-1} \times 100 \%
$$

where $\widetilde{C}_{m}$ denotes the fuzzy value of maximum ground-level concentration of the $m$ - th pollutant, and $C_{0 m}$ is the standard concentration value of ambient air quality
(Ministry of Environmental Protection of the PRC, 2008).

(6) The standard evaluation index $\widetilde{c}_{3}$ of major pollution factors of underground water is used to estimate the impact on groundwater due to CBM project. The major pollution factors include each pollutant (water quality factor) whose ratio of accumulative pollution load is over $70 \%$. $\widetilde{c}_{3}$ could be computed as follows:

$$
\widetilde{c}_{3}=\max _{k}\left\{\widetilde{Q}_{k}\left(Q_{s k}\right)^{-1}\right\}
$$

where $\widetilde{Q}_{k}$ denotes the fuzzy mass concentration of the $k$ - th major pollution factor and $\widetilde{Q}_{s k}$ denotes the corresponding standard mass concentration(Ministry of Environmental Protection of the PRC, 2008). $\widetilde{c}_{3}>1$ indicates that the monitoring value is over the stipulated one, and the larger value means the severer pollution.

(7) The Nemero pollution index $\widetilde{c}_{4}$ of soil is used to estimate the comprehensive environmental quality which is computed using the following equation:

$$
\widetilde{c}_{4}=\left[\frac{\left(\widetilde{C}_{z}^{\prime} / S_{z}\right)_{\max }^{2}+\left(\widetilde{C}_{z}^{\prime} / S_{z}\right)_{\text {ave }}^{2}}{2}\right]^{-2}
$$

where $\widetilde{C}_{z}^{\prime}$ stands for the fuzzy value of heavy metal content in soil, $S_{z}$ denotes the standard value of soil environmental quality, $\left(\widetilde{C}_{z}^{\prime} / S_{z}\right)_{\max }$ and $\left(\widetilde{C}_{z}^{\prime} / S_{z}\right)_{\text {ave }}$ indicates the maximum and average fuzzy values of a certain heavy mental pollutant respectively.

(8) Percentage $\widetilde{c}_{5}$ of forestry and grass 
coverage is designed to estimate the situation of soil and water conservation and ecological functions, which is computed by the formula:

$$
\widetilde{e}_{5}=\widetilde{S}_{F G}\left(S_{T}\right)^{-1}
$$

where $\widetilde{S}_{F G}$ denotes the fuzzy total area of vegetation, and $S_{T}$ denotes the affected area due to CBM project.

(9) Exposure degree $\tilde{b}_{1}$ of $\mathrm{CBM}$ resource is computed by

$$
\widetilde{b}_{1}=\widetilde{Q}_{M}\left(\widetilde{Q}_{A}\right)^{-1}
$$

where $\widetilde{Q}_{M}$ denotes the fuzzy value of exploitation quantity $\mathrm{CBM}$, and $\widetilde{Q}_{A}$ denotes the fuzzy value of CBM recoverable reserves.

(10) Risk prevention ability $\tilde{b}_{2}$ of personnel is to reflect the anti-risk and adaptive capabilities of hazard-affective bodies (personnel), including the employees' professional quality, the situation of risk prevention skills training and the standardization of emergency plan, which are described by means of language values: good, medium, bad. Then $\widetilde{b}_{2}$ is obtained in the same way as $\widetilde{e}_{1}$.

(11) Investment proportion $\widetilde{b}_{3}$ of prevention construction funds reflects the anti-risk and adaptive capabilities of hazard-affected bodies (property), which is defined by

$$
\widetilde{b}_{3}=\widetilde{F}_{R P}\left(\widetilde{G}_{T C}\right)^{-1}
$$

where $\widetilde{F}_{R P}$ denotes the ratio of risk prevention funds and $\widetilde{G}_{T C}$ is the total construction funds.

(12) Proportion $\tilde{l}_{1}$ of economic loss reflects the economic loss caused by eco-environmental risk, which is calculated by

$$
\widetilde{l}_{1}=\left(\widetilde{F}_{D E L}+\widetilde{F}_{I E L}\right)\left(\widetilde{G}_{O V}\right)^{-1}
$$

where $\widetilde{F}_{D E L}$ is the direct economic loss, $\widetilde{F}_{I E L}$ is the indirect economic loss and $\widetilde{G}_{O V}$ is the gross output value.

(13) Incidence $\widetilde{l}_{2}$ of occupational diseases indicates the injury caused by nonvital but persistent disaster losses, which is calculated by

$$
\tilde{l}_{2}=\tilde{P}_{O D}\left(\tilde{P}_{W}\right)^{-1}
$$

where $\widetilde{P}_{O D}$ is the number of patients with occupational disease and $\widetilde{P}_{W}$ is the total numbers of people in CBM production activities.

(14) Casualty rate $\tilde{l}_{3}$ reflects the damage and loss caused by abrupt momentous injury accidents which is calculated by

$$
\widetilde{l}_{3}=\widetilde{P}_{C F}\left(\widetilde{P}_{W}\right)^{-1}
$$

where $\widetilde{P}_{\mathrm{CF}}$ is the number of fatalities due to the pollution or destructive accident and $\widetilde{P}_{W}$ is the same as in Formula (15) .

\section{Construction of Comprehensive}

\section{Assessment Model}

Based on the fuzzy quantization in Section 3, by inputting fuzzy rules and training samples obtained from expert system into Mamdani intuitionistic fuzzy neural networks (MIFNN) using comprehensive generated method (Lei et al, 2014) and by adjusting the learning algorithm of the model, we can get the actual outputs of eco-environmental risk assessment in CBM industrialization development.

\subsection{Model structure}

The MIFNN model is a fuzzy multilayer feed forward neural network system with multi-input and single-output structure. By referring to the hierarchical structure of self-organizing intuitionistic fuzzy neural network model proposed by Xu et al (2010) and Lei et al (2014) and combining with fuzzy control theory, the MIFNN model can be described as a six-layer networks model as shown in Fig.3.

The first layer, the layer for input variables, inputs variable values into the fuzzy neural system. Owing to the fuzzifier in Mamdani model, the input values should be the exact values $\left(e_{1}, \cdots, l_{3}\right)^{T}$.

The second layer, the layer for fuzzy linguistic variables, is designed for inputting attribute functions (membership functions and non-membership functions). By Formula (4) through (6), we denote the attribute functions by interval-valued Gaussian membership functions, and the output values gained by each node are interval-valued fuzzy sets $\left[\mu_{i j}^{-}, \mu_{i j}^{+}\right]$. Then the vector of the fuzzy sets $\left(\widetilde{e}_{1}, \cdots, \widetilde{l}_{3}\right)^{T}$ is obtained. 


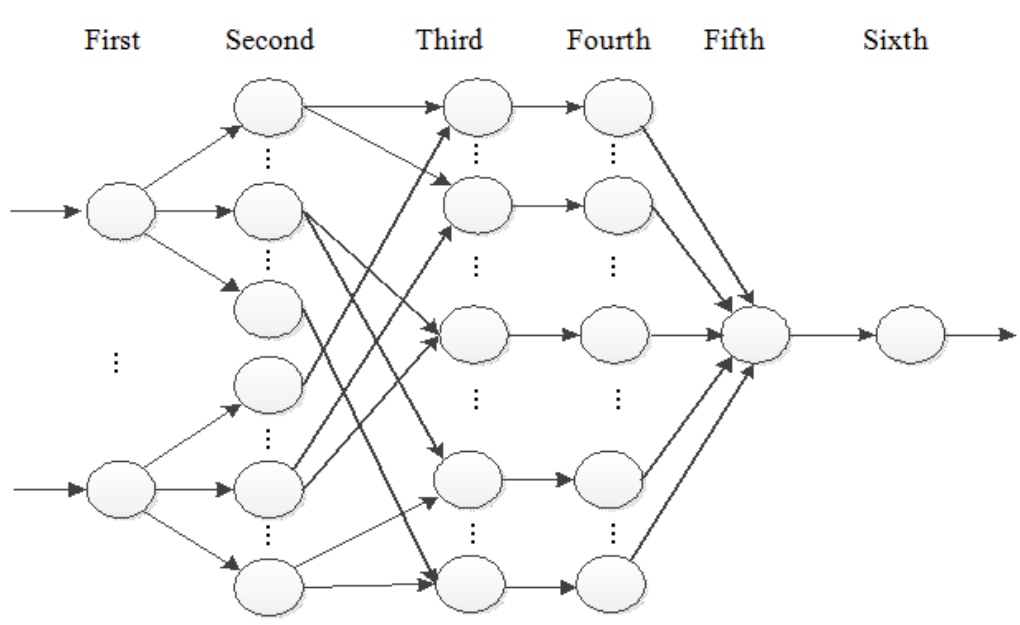

Fig. 3. Mamdani Intuitionistic Fuzzy Neural Network Model.

The third layer, the layer for inference rules, is designed for marching the antecedent of interval-valued fuzzy rules. Each node in this layer represents an antecedent of the fuzzy rules, and the output value of the $j$ th node is $\tilde{\Phi}_{i}=\left|\varphi_{i}^{-}, \varphi_{i}^{+}\right|$. Meanwhile, in order to reduce the information loss, we replace the $\wedge$ and $\vee$ in the model respectively by the addition and multiplication operations in real numbers.

$$
\varphi_{j}^{-}=\prod_{i=1}^{14} \mu_{i j}^{-}, \varphi_{j}^{+}=\prod_{i=1}^{14} \mu_{i j}^{+}
$$

The forth layer, the layer for output linguistic variables, outputs the consequent of fuzzy rules of each node in the upper layer. The output linguistic variable of the $j$ th node is the interval-valued fuzzy set:

$\widetilde{\omega}_{j}=\left[\omega_{j}^{-}, \omega_{j}^{+}\right]=\left[\mu_{i j}^{-}, \mu_{i j}^{+}\right] \cdot\left(\widetilde{e}_{1}+\cdots+\widetilde{l}_{3}\right)$

The fifth layer, the layer for output processing, is defuzzification for which the center average defuzzifier is utilized. The output values are $\widetilde{R}=\left[R^{-}, R^{+}\right]$and $R^{-}$and $R^{+}$are defined by

$$
R^{-}=\frac{\sum_{j=1}^{u} \varphi_{j}^{-} \omega_{j}^{-}}{\sum_{j=1}^{u} \varphi_{j}^{-}}, R^{+}=\frac{\sum_{j=1}^{u} \varphi_{j}^{+} \omega_{j}^{+}}{\sum_{j=1}^{u} \varphi_{j}^{+}}
$$

where $u$ is the number of fuzzy rules.

The sixth layer, the layer for output results, shows the risk assessment value $R_{C B M}$ of CBM industrialization development, based on the intuitionistic fuzzy sets theory of interval values and the defuzzification of center average defuzzifier. The exact value $R_{C B M}$, is the satisfactory stack result of the input information from the risk assessment indexes in the network system.

$$
R_{C B M}=\frac{R^{-}+R^{+}}{2}
$$

After the processing of six layers of network structure in the MIFNN model, we obtain $R_{C B M}$ as

$$
R_{C B M}=\frac{\sum_{j=1}^{u} \prod_{i=1}^{14} \mu_{i j}^{-}\left(\sum_{i=1}^{14} \mu_{i j}^{-} x_{i}\right)}{2 \sum_{j=1}^{u} \prod_{i=1}^{14} \mu_{i j}^{-}}+\frac{\sum_{j=1}^{u} \prod_{i=1}^{14} \mu_{i j}^{+}\left(\sum_{i=1}^{14} \mu_{i j}^{+} x_{i}\right)}{2 \sum_{j=1}^{u} \prod_{i=1}^{14} \mu_{i j}^{+}}
$$

Meanwhile, according to the universal approximation theory, there must be a Gaussian fuzzy logic system, in which any given function can be approached with any precision. The global approximation of MIFNN model can be readily verified.

\subsection{Self-adaptive learning algorithm of the model}

With the MIFNN model for eco-environmental risk assessment of CBM industrilization development, the correspondence between risk assessment indexes and the real output value $R_{C B M}$ is obtained after some repeated learning training.

Now assume that the desired output value is $R$. Then the squared error function is $E_{P}=\frac{\left(R_{C B M}-R\right)^{2}}{2}$. Substitute (5) and (6) into 
(21) to obtr ${ }^{\text {ATLANTIS }}$ PRESS isformed fuzzy system which possesses the curve center $\delta_{i j}$, the curve width $\sigma_{i j}$ (calculated by the average value of $\sigma_{i j}^{-}$and $\sigma_{i j}^{+}$) of Gaussian membership function and $\omega_{i j}$ ( calculated by the average value of $\omega_{i j}^{-}$and $\left.\omega_{i j}^{+}\right)$.

By the algorithm of first-order gradient search, the adjusted variables $\delta_{i j}, \sigma_{i j}$ and $\omega_{i j}$ can be expressed by

$$
\begin{aligned}
\delta_{i j}(n+1)-\delta_{i j}(n) & =-\eta \frac{\partial E_{P}}{\partial \delta_{i j}} \\
\sigma_{i j}^{-}(n+1)-\sigma_{i j}^{-}(n) & =-\eta \frac{\partial E_{P}}{\partial \sigma_{i j}^{-}} \\
\sigma_{i j}^{+}(n+1)-\sigma_{i j}^{+}(n) & =-\eta \frac{\partial E_{P}}{\partial \sigma_{i j}^{+}} \\
\omega_{i j}^{-}(n+1)-\omega_{i j}^{-}(n) & =-\eta \frac{\partial E_{P}}{\partial \omega_{i j}^{-}} \\
\omega_{i j}^{+}(n+1)-\omega_{i j}^{+}(n) & =-\eta \frac{\partial E_{P}}{\partial \omega_{i j}^{+}}
\end{aligned}
$$

Then, we can obtain the calibrated output surface.

\section{Case Study}

In this section, we utilize the sample data obtained by laboratory simulation to analyze the reasonability feasibility and superiority of the MIFNN model in Section 4.

\subsection{Data sources and preprocessing}

Our data come from the corresponding scenario simulations and experts' experiences. The fuzzy rules and training samples are obtained from the comprehensive generated method. The input-output relation surfaces are obtained after repeated learning trainings by Matlab software, which are shown in Fig. 4. These 3-dimensional relation surfaces are relatively smooth, which verifies the reasonability of fuzzy rules.

\subsection{Results analysis}

Now we analyze the simulation results of the model through 50 groups of cases which are

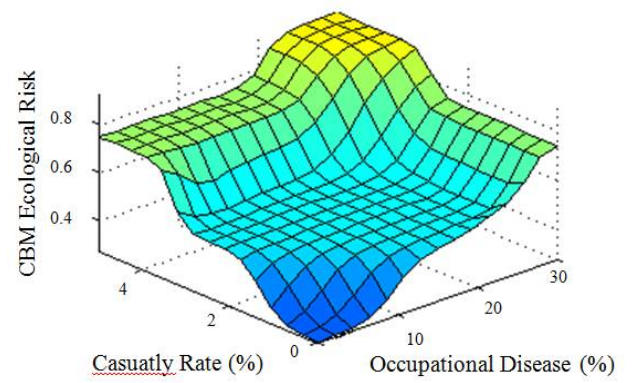

(a) Ecological Risk-occupational Disease-casualties

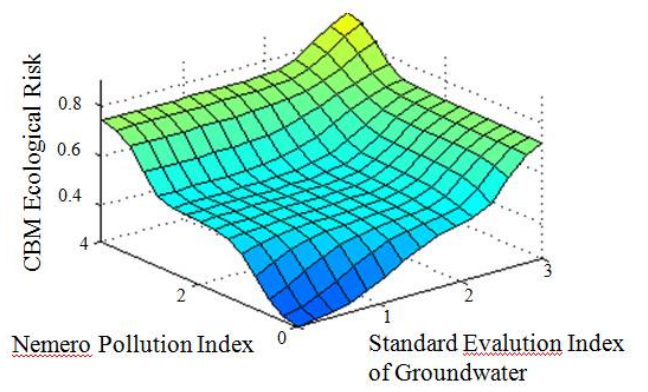

(b) Ecological Risk-groundwater-soil Contamination

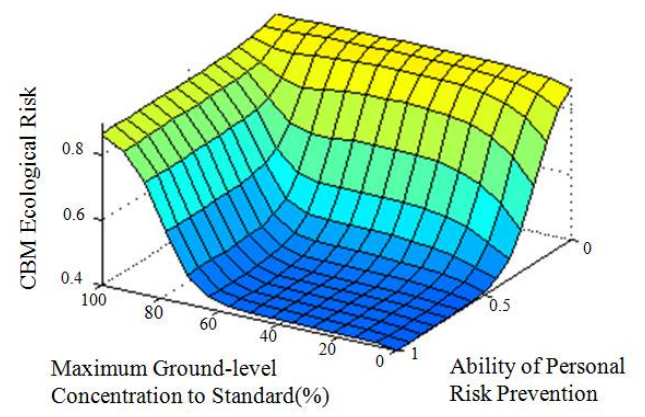

(c) Ecological Risk-personnel Prevention-air

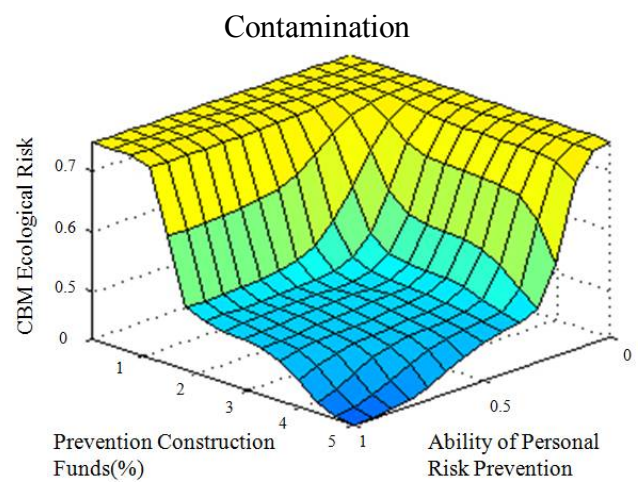

(d) Ecological Risk- precaution Funds-personnel Prevention

Fig. 4. Relation Surfaces

selected randomly and partial input and output values are shown in Table 3. Take the No.3 group of data in Table 3 as an example. The attribute characteristics of eco-environmental risk in CBM industrialization development in this group are: the hydrogeological condition is complex (the stability of coal seam is medium, 
the vulnerab ${ }_{\text {PRESS }}^{\text {ATLANTIS }}$ if medium, and the sensitivity degree of the groundwater environment is sensitive); the degree of mechanization is high (the utilization rate of mechanical equipments is high, the mine comprehensive yield of CBM is high, and the informationization degree of management is low); the average failure rate of mechanical equipments is $6.25 \times 10^{-4} h^{-1}$, and they can perform production tasks normally within $7 \times 24 h$; the impact of bad weather is low (the damage degree is weak, the time of duration is medium, and the affected area is small); the ratio of the maximum ground-level concentration to the standard concentration of major air pollutants is $73 \%$; the standard index evaluation of groundwater major pollution factors is 1.15; the Nemero pollution index evaluation of soil is 1.45; the percentage of forestry and grass coverage is $18.3 \%$; the resource exposure degree of $\mathrm{CBM}$ is $23.3 \%$; the ability of personal risk prevention is good (the employees' professional quality is medium, the situation of risk prevention skills training is good, and the standardization of emergency plan is medium); the investment proportion of prevention construction funds is $1.78 \%$; the proportion of economic loss is $2.65 \%$; the incidence of occupational diseases is $6.1 \%$; and the casualty rate is $0.96 \%$. Calculated with the MIFNN model, the risk assessment value is 0.4574 , which is the medium risk level as anticipated, which indicates that the eco-environment is mildly contaminated, and the situation is manageable and can be controlled. The administrators should strength the supervision and precaution.

Table. 3. The Input and Output Values of Attribute Parameters in CBM Eco-environmental Risk

\begin{tabular}{c|c|c|c|c|c|c|c|c|c|c|c|c|c|c|c}
\hline Num. & $e_{1}$ & $e_{2}$ & $e_{3}$ & $c_{1}$ & $c_{2}$ & $c_{3}$ & $c_{4}$ & $c_{5}$ & $b_{1}$ & $b_{2}$ & $b_{3}$ & $l_{1}$ & $l_{2}$ & $l_{3}$ & Outputs \\
\hline 1 & 0.83 & 0.67 & $e^{-0.12}$ & 0.33 & 39 & 0.86 & 0.67 & 21.5 & 13.3 & 0.50 & 2.52 & 1.76 & 4.33 & 0.64 & 0.2639 \\
2 & 0.83 & 0.67 & $e^{-0.18}$ & 0.33 & 80 & 1.27 & 1.52 & 16.4 & 36.7 & 0.67 & 1.01 & 2.96 & 9.96 & 1.63 & 0.5215 \\
3 & 0.67 & 0.67 & $e^{-0.11}$ & 0.17 & 73 & 1.15 & 1.45 & 18.3 & 23.3 & 0.67 & 1.78 & 2.65 & 6.10 & 0.96 & 0.4574 \\
4 & 0.83 & 0.50 & $e^{-0.32}$ & 0.50 & 86 & 1.48 & 1.83 & 7.7 & 33.3 & 0.33 & 0.94 & 3.83 & 8.29 & 1.41 & 0.6605 \\
5 & 0.50 & 0.83 & $e^{-0.03}$ & 0.17 & 57 & 0.76 & 0.95 & 23.9 & 16.7 & 0.83 & 2.62 & 1.85 & 5.53 & 0.92 & 0.1517 \\
$\ldots$ & $\ldots$ & $\ldots$ & $\ldots$ & $\ldots$ & $\ldots$ & $\ldots$ & $\ldots$ & $\ldots$ & $\ldots$ & $\ldots$ & $\ldots$ & $\ldots$ & $\ldots$ & $\ldots$ & $\ldots$ \\
46 & 0.83 & 0.67 & $e^{-0.06}$ & 0.33 & 55 & 1.12 & 1.72 & 13.9 & 26.7 & 0.67 & 2.03 & 2.61 & 5.31 & 0.90 & 0.4050 \\
47 & 0.67 & 0.67 & $e^{-0.19}$ & 0.33 & 87 & 2.35 & 3.01 & 6.6 & 50.0 & 0.33 & 0.82 & 6.17 & 13.22 & 2.19 & 0.8982 \\
48 & 0.83 & 0.67 & $e^{-0.21}$ & 0.50 & 63 & 0.93 & 0.97 & 15.3 & 23.3 & 0.50 & 2.41 & 2.35 & 6.39 & 1.06 & 0.3046 \\
49 & 0.50 & 0.83 & $e^{-0.17}$ & 0.33 & 45 & 0.82 & 0.86 & 16.5 & 16.7 & 0.50 & 2.37 & 2.23 & 5.28 & 0.89 & 0.2797 \\
50 & 0.83 & 0.50 & $e^{-0.32}$ & 0.67 & 92 & 1.89 & 2.23 & 9.8 & 40.0 & 0.17 & 0.56 & 4.85 & 10.35 & 1.74 & 0.7992 \\
\hline
\end{tabular}

\subsection{Comparative analysis with the FNN model}

In order to illustrate the superiority of the MIFNN model, we compare it with the fuzzy neural networks (FNN) model. To increase the comparability, we employ the simulation cases in Table 3 and apply the FNN model. The comparative analysis is based on the upper and lower bounds of the interval values in formula (4)-(5).

Fig.5 shows that both the MIFNN and
FNN models could reflect the level of eco-environmental risk assessment in general. However, some values obtained by the FNN model are out of the interval values of intuitionistic fuzzy sets, e.g. the data in the No.13 and No.41 groups. In view of this, the further analysis for the evaluation quality of the both models is needed. Calculate respectively the degree of dispersion $\left(\sigma_{\mathrm{MIFNN}}, \sigma_{\mathrm{FNN}}\right)$ and the coefficient of variation ( $C V_{\mathrm{MIFNN}}, C V_{\mathrm{FNN}}$ ) which are shown in Table 4. 


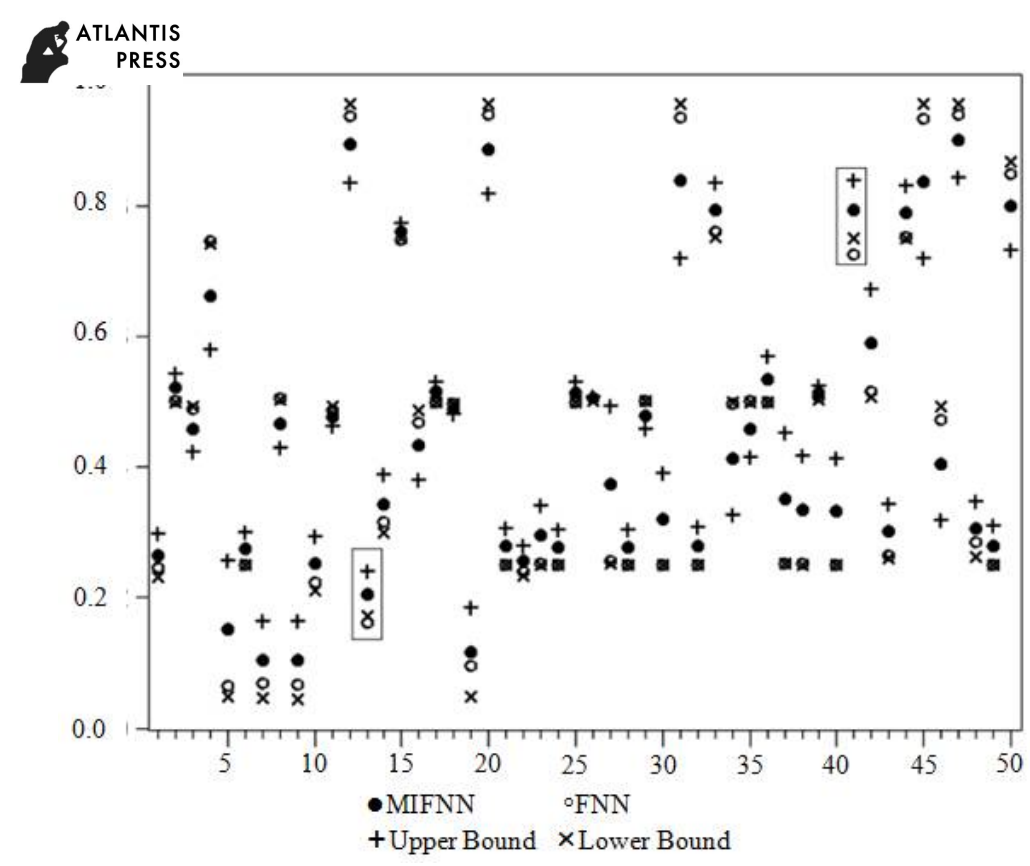

Fig. 5. The Comparison of Inference Results Between MIFNN and FNN.

Table. 4. The Evaluation Comparison of MIFNN and FNN

\begin{tabular}{c|c|c|c|l}
\hline & MIFNN & FNN & $\begin{array}{c}\text { Numeric } \\
\text { comparison }\end{array}$ & \multicolumn{1}{c}{ Result analysis } \\
\hline$\sigma$ & 0.0507 & 0.0646 & $\sigma_{M F N N}<\sigma_{F N N}$ & $\begin{array}{l}\text { The stability of MIFNN model is superior to FNN } \\
\text { model } \\
\text { The accuracy of MIFNN model is superior to FNN } \\
\text { model }\end{array}$ \\
\hline
\end{tabular}

It can be seen from Fig. 5 and Table 4 that the stability and accuracy of the MIFNN model are superior. Therefore, it is more suitable for the eco-environmental risk assessment in CBM industrialization development.

\section{Conclusions}

This paper provides a method of eco-environ -mental risk assessment during CBM industrialization development based on the MIFNN model with both nonlinearity and global-approximation characteristics, by optimizing the index system and utilizing Atanassov intuitionistic fuzzy sets to improve data quality. The rationality and validity of the model are verified by simulation cases. It can be concluded that the expected risk level of eco-environment is in conformity with the assessment results. The comparative analysis indicates that both the MIFNN and FNN models are effective in assessing the risk level of eco-environment; however, the former model is superior to the latter as far as stability and accuracy are concerned. Therefore the application of the MIFNN model on empirical study will provide a decision basis for ecological supervision and risk precaution and management. Nevertheless, the number of attribute indexes in the model is relatively large, which results in some defects on precision, though we effectively adjust parameters in fuzzy rules and training samples. Therefore, we will further improve the risk assessment model by trimming and optimizing fuzzy inference rules and taking multidisciplinary complementary advantages in future. Meanwhile, we will develop evaluation software to implement the computer management of eco-environmental risk assessment on CBM industrialization development.

\section{Acknowledgements}

This work was supported by the National Science Foundation for Young Scholars of China (Grant No. 41101507); the National Science Foundation of China (Grant No. 71373170); the 
Program for ${ }_{\text {PRESS }}^{\text {ATLANTIS }}$ phy and Social Sciences

Research of Higher Learning Institutions of Shanxi (PSSR), China (Grant No. 2017314); the Program for the Top Young Academic Leaders of Higher Learning Institutions of Shanxi (TYAL), China; and by the Soft science Foundation of Shanxi Province, China (Grant No. 2017041025 $-2)$.

\section{References}

J. Wang, Irrigation effects on soil and plant by using coal bed mathane produced water in Qinshui basin, China University of Mining \& Technology, (2014)

H. H. Liu, S. X. Sang, L. W. Cao, et al. Heavy metal pollution feature analysis and potential ecological risk assessment of the coalbed methane production on the topsoil quality of the mining area, Journal of Safety and Environment, (3) (2014) 288-293.

L. W. Hu, H. X. Chen, W. G. Yang, Study on Environmental Impact and Management of Coal Bed Methane Development, Environmental Protection Science, (6) (2009) 51-54.

Y. Y. Li, J. W. Kang, Analysis of potential ecological influence during grounding mining of coal bed methane, China Coal, (3) (2014) 24-127.

Y. Song, X. M. Zhang, S. B. Liu, et al. Basic Theory of Coalbed Methane Geology and Exploitation in China, Science Press, (2012).

J. G. Li, Study on simulation and risk Assessment of leakage and diffusion of coalbed methane field gathering and transportation, China University of Mining \& Technology, (2014).

J. A. Beckstrom, D. G. Boyer, Aquifer-protection considerations of coalbed methane development in the San-Juan Basin, Society of Petroleum Engineers Formation Evaluation, 8(1) (1993) 71-79.

D. Chafin, D. Swanson, D. Gery, Methane-concentration and methane-isotope data for groundwater and soil gas in the Animas River Valley. Colorado and New Mexico, (1996).

US Environmental Protection Agency. Study design for evaluating of impacts to underground sources of drinking water by hydraulic fracturing of coalbed methane reservoirs. Washington DC, 2001.

US Environmental Protection Agency. Evaluation of impacts to underground sources of drinking water by hydraulic fracturing of coalbed methane reservoirs. Washington DC, 2004.

K. Cheung, H. Sanei, P. Klassen, et al. Produced fluids and shallow groundwater in coalbed methane (CBM) producing regions of Alberta, Canada: Trace element and rare earth element geochemistry, International Journal of Coal Geology, 77 (2009) 338-349.

K. Cheung, P. Klassen, B. Mayer, et al. Major ion and isotope geochemistry of fluids and gases from coalbed methane and shallow groundwater wells in Alberta, Canada, Applied Geochemistry, 25 (2010) 1307-1329.

K. J. Dahm, C. M. Van Straaten, J. Munakata-Marr, et al. Identifying well contamination through the use of $3 \mathrm{D}$ fluorescence spectroscopy to classify coalbed methane produced water, Environmental Science and Technology, 47 (2013) 649-656.

R. F. Aguilera, R. D. Ripple, R. Aguilera, Link between endowments, economics and environment in conventional and unconventional gas reservoirs, Fuel, 126 (2014) 224-238.

Y. T. Pei, L. K. Zhang, J. K. He, et al. Ecological effect evaluation of CBM development based on PSR framework model, Environmental Protection of Oil \& Gas Fields, (1) (2015) 25-27.

W. Sun, Y. Xue, X. J. Ren, The index system of ecological risk assessment based on the industrialization development of coal-bed methane. In: Huang, C.F. Emerging Economies, Risk and Development, and Intelligent Technology: Proceedings of the 5th International Conference on Risk Analysis and Crisis Response. Morocco: CRC Press, (2015) 495-502.

P. J. Shi, Theory and practice on disaster system research in a fifth time, Journal of Natural Disasters, (5) (2009) 1-9.

Y. Xue, The Study of Soft Hierarchical Model on Integrated Risk Assessment for Natural Disaster. Meteorological Press, (2014).

K. Atanassov, Intuitionistic fuzzy sets, Fuzzy Set Sys, 20(1) (1986) 87-96.

X. H. Yuan, H. X. Li, J. J. Song, Interval-valued level cut sets, decomposition theorems and representation theorems on interval-valued fuzzy sets. Fuzzy Systems and Mathematics, (5) (2013) $1-10$.

H. P. Xie, J. H. Wang, B. H. Shen, et al. New idea of coal mining: scientific mining and sustainable mining capacity, Journal of China Coal Society, (7) (2012) 1069-1079.

J. Feng, Q. Sun, P. C. Luo, et al. Equipment Reliability and Comprehensive Security, National University of Defense Technology Press, Changsha, Hunan, China, (2008).

China's ministry of environmental protection. Guidelines for Environmental Impact Assessment: Atmospheric Environment. Environmental Science Press, Beijing, China, 2008.

Y. J. Lei, J. Zhao, Y. L. Lu, et al. Intuitionistic Fuzzy Set Theory and Application. Science Press, Beijing, China, (2014).

X. L. Xu, Y. J. Lei, W. B. Xie, Self-organising intuitionistic fuzzy neural networks based on UKF. Acta Electronica Sinica, (3) (2010) 638-645. 